\title{
Lyapunov-Like Conditions for the Existence of Zeno Behavior in Hybrid and Lagrangian Hybrid Systems
}

\author{
Andrew Lamperski and Aaron D. Ames
}

\begin{abstract}
Lyapunov-like conditions that utilize generalizations of energy and barrier functions certifying Zeno behavior near Zeno equilibria are presented. To better illustrate these conditions, we will study them in the context of Lagrangian hybrid systems. Through the observation that Lagrangian hybrid systems with isolated Zeno equilibria must have a onedimensional configuration space, we utilize our Lyapunov-like conditions to obtain easily verifiable necessary and sufficient conditions for the existence of Zeno behavior in systems of this form.
\end{abstract}

\section{INTRODUCTION}

Zeno behavior occurs in a hybrid system if an infinite number of discrete transitions occur in a finite amount of time. The goal of this paper is to study a specific type of Zeno behavior, namely, Zeno behavior that occurs near isolated Zeno equilibria. A Zeno equilibrium is a set of points in the continuous domains of a hybrid system that are invariant under the discrete dynamics (but not the continuous dynamics). Isolated Zeno equilibria form the Zeno analogue of isolated equilibria. Just as there can be complicated sets of equilibria in dynamical systems, there can be much more complicated sets of Zeno equilibria. Yet as the study of isolated equilibria of dynamical systems has been hugely successful, we believe that an important first step to the understanding of Zeno behavior is the detailed study of isolated Zeno equilibria.

The two main results of this paper are: Lyapunov-like sufficient conditions for existence of Zeno behavior near isolated equilibria, and a characterization of Zeno behavior near isolated Zeno equilibria of Lagrangian hybrid systems.

Unlike classical Lyapunov theory, our Lyapunov-like conditions for the existence of Zeno behavior actually involve two functions on each domain. The first function is used in a manner similar to standard Lyapunov functions, i.e., it is used to prove positive invariance of compact sets around our Zeno equilibria. The second function is a barrier function [1] used to capture explicit information about how long an execution spends in a continuous domain. In addition, these functions appear to be searchable via polynomial optimization [2], [3]. Therefore, this result works toward the goal of automated analysis of hybrid systems.

The second main result presented in this paper gives necessary and sufficient conditions for Zeno behavior near isolated Zeno equilibria of Lagrangian hybrid systems, which model mechanical systems undergoing impacts. To obtain

A. Lamperski and A. D. Ames are with Control and Dynamical Systems Department, California Institute of Technology, Pasadena, CA 91125 $\{$ andyl, ames\}ecds.caltech.edu these conditions, we first show that Lagrangian hybrid systems with isolated Zeno equilibria must necessarily have a one-dimensional configuration space. This simple structure allows us to explicitly construct the desired Lyapunovlike functions that prove the existence of Zeno behavior. Moreover, due to the explicit nature of these functions, we are able to demonstrate that one can check for the existence and nonexistence of Zeno behavior by evaluating functions given in the system description at a single point. This result represents, to the authors' knowledge, the only known necessary and sufficient result for Zeno behavior in uncontrolled hybrid systems. Necessary and sufficent conditions for Zeno behavior in a significantly different class of controlled hybrid systems were found in [4].

Although results presented in this paper make gains toward a better understanding of Zeno behavior, they also indicate possible future research directions. The most compelling of these is motivated by the fact that isolated Zeno equilibria only occur in Lagrangian hybrid systems with onedimensional configuration spaces, indicating that to understand Zeno behavior in Lagrangian hybrid systems, more complicated sets of Zeno equilibria must be studied. Also, due to the Lyapunov-like nature of the sufficient conditions presented, the hope is that in the future these conditions could be automated. Finally, the Lyapunov-like conditions given in this paper also imply that Zeno executions converge to the Zeno equilibria; that is, Zeno behavior is closely related to the stability of Zeno equilibria. This relationship was first noticed in [5], where the asymptotic stability of Zeno equilibria was studied; in fact, the Lyapunov-like conditions presented in that work are very similar to the conditions presented in this paper. Yet, the claim made in [5] that the asymptotic stability of Zeno equilibria implies the existence of Zeno behavior has since proven to be incorrect. Therefore, the connection between the stability of Zeno equilibria and Zeno behavior still remains to be made, although this paper provides strong evidence for the existence of such a connection.

Due to the subtle and complex nature of Zeno behavior, it has been studied in many forms and from many different perspectives. Most of the conditions for Zeno behavior are necessary and tend to be very conservative; see [6], [7], [8] for general hybrid systems, and [9], [10] for linear complementarity systems. Obtaining sufficient conditions for the existence of Zeno behavior seems to be a much more challenging task. Therefore, very few such conditions have been given to date and those that have been obtained apply to very limited classes of hybrid systems [11]. We also 
note that this paper studies Zeno behavior in Lagrangian hybrid systems, which were studied in [12], [13] and [14] as motivated by [15]. Finally, the characterization of Zeno behavior presented in this paper complements the topological characterization of Zeno behavior presented in [16].

\section{HybRid SYSTEMS \& ZeNo EQUILIBRIA}

In this section, we introduce the basic notations on which the rest of the paper will build. That is, we define hybrid systems, executions, Zeno equilibria and convergence to Zeno equilibria.

Definition 1: A hybrid system on a cycle is a tuple:

$$
\mathscr{H}=(\Gamma, D, G, R, F),
$$

where

- $\Gamma=(Q, E)$ is a directed cycle, with

$$
\begin{aligned}
Q= & \left\{q_{0}, \ldots, q_{k-1}\right\}, \\
E= & \left\{e_{0}=\left(q_{0}, q_{1}\right), e_{1}=\left(q_{1}, q_{2}\right),\right. \\
& \left.\ldots, e_{k-1}=\left(q_{k-1}, q_{0}\right)\right\} .
\end{aligned}
$$

We denote the source of an edge $e \in E$ by source(e) and the target of an edge by target $(e)$.

- $D=\left\{D_{q}\right\}_{q \in Q}$ is a set of domains, where $D_{q}$ is a smooth manifold.

- $G=\left\{G_{e}\right\}_{e \in E}$ is a set of guards, where $G_{e} \subseteq$ $D_{\text {source }(e)}$ is and embedded submanifold of $D_{\text {source }(e)}$.

- $R=\left\{R_{e}\right\}_{e \in E}$ is a set of reset maps, where $R_{e}: G_{e} \subseteq$ $D_{\text {source(e) }} \rightarrow D_{\text {target }(e)}$ is a smooth map.

- $F=\left\{f_{q}\right\}_{q \in Q}$, where $f_{q}: D_{q} \rightarrow T D_{q}$ is a Lipschitz vector field on $D_{q}$.

Remark 1: Note that the motivation for considering hybrid systems on cycles, rather than hybrid systems on general graphs, is that cycles are indicative of Zeno behavior (see [6] and $[8]$ ). Therefore, beginning with hybrid systems defined on cycles will greatly simplify our analysis, while still capturing characteristic types of Zeno behavior.

Definition 2: An execution of a hybrid system $\mathscr{H}=$ $(\Gamma, D, G, R, F)$ is a tuple:

$$
\chi=(\Lambda, I, \rho, C)
$$

where

- $\Lambda=\{0,1,2, \ldots\} \subseteq \mathbb{N}$ is a finite or infinite indexing set,

- $I=\left\{I_{i}\right\}_{i \in \Lambda}$ where for each $i \in \Lambda, I_{i}$ is defined as follows: $I_{i}=\left[\tau_{i}, \tau_{i+1}\right]$ if $i, i+1 \in \Lambda$ and $I_{N-1}=$ $\left[\tau_{N-1}, \tau_{N}\right]$ or $\left[\tau_{N-1}, \tau_{N}\right)$ or $\left[\tau_{N-1}, \infty\right)$ if $|\Lambda|=N$, $N$ finite. Here, for all $i, i+1 \in \Lambda, \tau_{i} \leq \tau_{i+1}$ with $\tau_{i}, \tau_{i+1} \in \mathbb{R}$, and $\tau_{N-1} \leq \tau_{N}$ with $\tau_{N-1}, \tau_{N} \in \mathbb{R}$.

- $\rho: \Lambda \rightarrow Q$ is a map such that for all $i, i+1 \in \Lambda$, $(\rho(i), \rho(i+1)) \in E$. This is the discrete component of the execution.

- $C=\left\{c_{i}\right\}_{i \in \Lambda}$ is a set of continuous trajectories, and they must satisfy $\dot{c}_{i}(t)=f_{d(i)}\left(c_{i}(t)\right)$ for $t \in I_{i}$.
We require that when $i, i+1 \in \Lambda$,

$$
\begin{gathered}
c_{i}(t) \in D_{\rho(i)} \forall t \in I_{i} \\
c_{i}\left(\tau_{i+1}\right) \in G_{(\rho(i), \rho(i+1))} \\
R_{(\rho(i), \rho(i+1))}\left(c_{i}\left(\tau_{i+1}\right)\right)=c_{i+1}\left(\tau_{i+1}\right) .
\end{gathered}
$$

When $i=|\Lambda|-1$, we still require that (i) holds.

Note that the continuous initial condition of an execution $\chi$ is given by $c_{0}\left(\tau_{0}\right) \in D_{\rho(0)}$. The discrete initial condition is given by $\rho(0)$.

The object of study in this paper will be Zeno executions, which are defined in the following manner:

Definition 3: An execution $\chi$ is Zeno if $\Lambda=\mathbb{N}$ and

$$
\lim _{i \rightarrow \infty} \tau_{i}=\tau_{0}+\sum_{i=0}^{\infty} \tau_{i+1}-\tau_{i}=\tau_{\infty}
$$

for some finite $\tau_{\infty} \in \mathbb{R}$.

A hybrid system $\mathscr{H}$ is Zeno $^{1}$ if there exists a Zeno execution $\chi$ such that $\tau_{i+1}-\tau_{i} \neq 0$ for some $i \in \mathbb{N}$.

Zeno behavior can be likened to stability, in that both types of behavior involve convergence. This motivates the study of the type of equilibria associated to Zeno behavior: Zeno equilibria. For more on Zeno behavior and Zeno equilibria, see [12].

Definition 4: A Zeno equilibria of a hybrid system $\mathscr{H}=$ $(\Gamma, D, G, R, F)$ is a set $z=\left\{z_{q}\right\}_{q \in Q}$ satisfying the following conditions for all $q \in Q$ :

- For the unique edge $e=\left(q, q^{\prime}\right) \in E$

$$
\begin{aligned}
& \quad-z_{q} \in G_{e}, \\
& \quad-R_{e}\left(z_{q}\right)=z_{q^{\prime}}, \\
& \text { - } f_{q}\left(z_{q}\right) \neq 0 .
\end{aligned}
$$

A Zeno equilibrium $z=\left\{z_{q}\right\}_{q \in Q}$ is isolated if there are neighborhoods $W_{q} \subset D_{q}, q \in Q$, such that there does not exist another Zeno equilibrium $\hat{z}=\left\{\hat{z}_{q}\right\}_{q \in Q}$ with $\hat{z}_{q} \in W_{q}$ for all $q \in Q$.

Note that, in particular, the conditions given in Definition 4 imply that for all $i \in\{0, \ldots, k-1\}$,

$$
R_{e_{i-1}} \circ \cdots \circ R_{e_{0}} \circ R_{e_{k-1}} \circ \cdots \circ R_{e_{i}}\left(z_{i}\right)=z_{i} .
$$

That is, the element $z_{i}$ is a fixed point under the reset maps composed in a cyclic manner.

Definition 5: An execution $\chi$ with $\Lambda=\mathbb{N}$ converges to a zeno equilibrium $z=\left\{z_{q}\right\}_{q \in Q}$ if given any neighborhoods $W_{q}$ with $z_{q} \in W_{q}$ for all $q \in Q$, there exists $j \in \Lambda$ such that $c_{k}(t) \in W_{\rho(k)}$ for all $k \geq j$ and all $t \in I_{k}$.

\section{LyAPUNOV-LiKe CONDITIONS FOR THE EXISTENCE OF ZENO BEHAVIOR}

This section presents Lyapunov-like sufficient conditions for the existence of Zeno behavior in hybrid systems on cycles. These results apply to a general class of hybrid

\footnotetext{
${ }^{1}$ The motivation for this definition is that we want to exclude the possibility that a hybrid system is "trivally" Zeno, i.e., the only Zeno executions are executions that begin at a Zeno Equilibria
} 
systems, and are fundamental in establishing the results presented in the Section V.

Conditions for Zeno behavior. We begin by introducing the conditions that will imply the existence of Zeno behavior. These involve conditions on the continuous and discrete portion of the hybrid system, together with the "convergence conditions" that indicate how executions will tend toward Zeno equilibria. As these conditions are, in some sense, analogous to stability conditions, it is not surprising that they involve Lyapunov-like functions. In fact, on each domain, they utilize two such functions-one is analogous to a standard Lyapunov function, while the other is analogous to a Barrier function [1] - that will interact through the "convergence conditions." Our conditions closely resemble the conditions given in [5] but avoid problems arising from slow convergence by enforcing geometric convergence in $\mathbf{C} 1$.

Let $\left\{z_{q}\right\}_{q \in Q}$ be a Zeno equilibrium of a hybrid system $\mathscr{H}, z_{q} \in W_{q} \subset D_{q}$ be open neighborhoods of $z_{q}$ for all $q \in Q, V_{q}, B_{q} \in C^{1}\left(W_{q}, \mathbb{R}\right)$ and $r<1, a>0$ and $b>$ 0 be constants. With this notation, consider the following conditions:

Continuous Conditions for Events:

EC1: $V_{q}(x)>0$ for all $x \in W_{q} \backslash\left\{z_{q}\right\}$ and $V_{q}\left(z_{q}\right)=0$ for all $q \in Q$.

EC2: $\dot{V}_{q}(x)=d V_{q}(x) \cdot f_{q}(x) \leq 0$ for all $x \in W_{q}$ and $q \in Q$.

EC3: $B_{q}(x) \geq 0$ for all $x \in W_{q}$ and $q \in Q$.

EC4: $\dot{B}_{q}(x)=d B_{q}(x) \cdot f_{q}(x)<0$ for all $x \in$ $W_{q}$, and $q \in Q$.

Discrete Condition for Events:

ED1: $V_{q^{\prime}}\left(R_{e}(x)\right) \leq V_{q}(x)$ for all $x \in G_{e} \cap W_{q}$, $q \in Q$ and the unique edge $e=\left(q, q^{\prime}\right)$.

Convergence Conditions:

C1: There exists $q \in Q$ such that $V_{q^{\prime}}\left(R_{e}(x)\right) \leq$ $r V_{q}(x)$ for all $x \in G_{e} \cap W_{q}$.

C2: $B_{q}(x) \leq b\left(V_{q}(x)\right)^{a}$ for all $x \in R_{\hat{e}}\left(G_{\hat{e}}\right) \cap$ $W_{q}, q \in Q$ and the unique edge $\hat{e}=\left(q^{\prime}, q\right)$.

With these conditions in hand, we now present the main result of this section.

Theorem 1: Let $\left\{z_{q}\right\}_{q \in Q}$ be an isolated Zeno equilibrium of a hybrid system $\mathscr{H}$. If conditions EC1-EC4, ED1, C1 and C2 hold, then there exists a neighborhood $Z_{q}$ of $z_{q}$ for all $q \in Q$ such that for all $x_{0} \in Z_{q}$ there exists a Zeno execution $\chi=(\Lambda, I, \rho, C)$ with $c_{0}\left(\tau_{0}\right)=x_{0}$. Therefore, $\mathscr{H}$ is Zeno.

We prove this theorem using two lemmas.

Lemma 1: Let $x(t)$ be the solution to $\dot{x}=f_{q}(x)$. If conditions EC1-EC4 hold, then there exists a compact set $\Omega_{q}^{\alpha} \subset W_{q}$, indexed by $\alpha>0$, and a positive constant $\gamma_{q}$ with the following properties:

- There exists an open set $Z_{q}$ such that $z_{q} \in Z_{q} \subset \Omega_{q}^{\alpha}$.

- There exists a time

$$
\tau \leq \frac{B_{q}(x(0))}{\gamma_{q}}<\infty
$$

such that $x(\tau) \in G_{e}$ when $x(0) \in \Omega_{q}^{\alpha}$, where here $e=\left(q, q^{\prime}\right)$.

Proof: Similar to Lyapunov's theorem, EC1 implies that when $\alpha>0$ is small enough, the connected component of the set:

$$
S_{q}^{\alpha}=\left\{x \in W_{q}: V_{q}(x) \leq \alpha\right\}
$$

containing $z_{q}$ is compact and contains a neighborhood of $z_{q}$. So we can restrict $W_{q}$ to a neighborhood $\bar{W}_{q}$ so that

$$
\Omega_{q}^{\alpha}=\left\{x \in \bar{W}_{q}: V_{q}(x) \leq \alpha\right\}
$$

is connected and compact. Further, $\Omega_{q}^{\alpha}$ contains a neighbor$\operatorname{hood} Z_{q}$ of $z_{q}$.

Since we have only a finite number of domains, we can pick $\bar{W}_{q}$ and a single $\alpha$ such that $\Omega_{q}^{\alpha}$ is connected and compact for each domain.

From EC2, if $x(0) \in \Omega_{q}^{\alpha}$, then $x(t) \in \Omega_{q}^{\alpha}$ for all $t \geq 0$ as long as $x(t) \in D_{q}$. That is, $x(t)$ remains in $\Omega_{q}^{\alpha}$ unless an event occurs.

Now we use EC3 and EC4 to show that events occur in finite time. Let

$$
-\gamma_{q}=\max _{x \in \Omega_{q}^{\alpha}} \dot{B}_{q}(x)
$$

Since $\Omega_{q}^{\alpha}$ is compact and $\dot{B}_{q}(x)<0$ for $x \in \Omega_{q}^{\alpha} \subset W_{q}$, we must have $\gamma_{q}>0$. So for $t \geq 0$

$B_{q}(x(t))=B_{q}(x(0))+\int_{0}^{t} \dot{B}_{q}(x(\sigma)) d \sigma \leq B_{q}(x(0))-\gamma_{q} t$.

Therefore, $x(t)$ must reach the guard within time $t^{*}=$ $B_{q}(x(0)) / \gamma_{q}$ by EC3.

Lemma 2: If conditions EC1-EC4 and ED1 hold, then for each $x_{0} \in \Omega_{q}^{\alpha}$, with $\alpha>0$ sufficiently small, there exists a unique execution $\chi=(\Lambda, I, \rho, C)$ with $c_{0}\left(\tau_{0}\right)=x_{0}, \rho(0)=$ $q$ and $\Lambda=\mathbb{N}$.

Proof: Continuity of $R_{e}$ and (ED1) imply that for $\alpha>$ 0 as in Lemma 1 and all $e=\left(q, q^{\prime}\right) \in E, R_{e}\left(G_{e} \cap \Omega_{q}^{\alpha}\right) \subset$ $\Omega_{q^{\prime}}^{\alpha}$

Let $c_{0}\left(\tau_{0}\right)=x_{0} \in \Omega_{q}^{\alpha}$. Assume without loss of generality that $q=q_{0}$. Then, since our hybrid system evolves on a directed cycle, conditions (i)-(iii) in (1) imply that

$$
\rho(i):=q_{\bar{i}}, \quad \bar{i}:=i \bmod |Q|
$$

So by construction, $c_{0}\left(\tau_{0}\right) \in \Omega_{\rho(0)}^{\alpha}$.

Now, inductively assume that $c_{i}\left(\tau_{i}\right) \in \Omega_{\rho(i)}^{\alpha}$ for some $i \geq 0$. From Lemma 1 , we can extend $c_{i}\left(\tau_{i}\right)$ to a solution $c_{i}(t)$ of $\dot{x}=f_{\rho(i)}(x)$ such that at some finite time $\tau_{i+1}$, we have $c_{i}\left(\tau_{i+1}\right) \in G_{e_{\bar{i}}} \cap \Omega_{\rho(i)}^{\alpha}$. From above, $R_{e_{\bar{i}}}\left(c_{i}\left(\tau_{i+1}\right)\right) \in$ $\Omega_{\rho(i+1)}^{\alpha}$. Therefore $c_{i}\left(\tau_{i}\right) \in \Omega_{\rho(i)}^{\alpha}$ implies that $c_{i+1}\left(\tau_{i+1}\right) \in$ $\Omega_{\rho(i+1)}^{\alpha}$.

The above argument holds inductively, so if $c_{0}\left(\tau_{0}\right) \in \Omega_{q_{0}}^{\alpha}$, then $c_{0}\left(\tau_{0}\right)$ extends to a unique execution $\chi=(\Lambda, I, \rho, C)$ with $\Lambda=\mathbb{N}$.

We will now use Lemmas 1 and 2 to prove Theorem 1

Proof: [of Theorem 1] Take $x_{0} \in \Omega_{q_{0}}^{\alpha}$. Let $\chi$ be the unique infinite execution with $c_{0}\left(\tau_{0}\right)=x_{0} \in \Omega_{q_{0}}^{\alpha}$. We show the total time spent in $D_{q_{0}}$ is finite. 
Repeated applications of $\mathbf{C} 1$ show that

$$
V_{q_{0}}\left(c_{j|Q|}\left(\tau_{j|Q|}\right)\right) \leq r^{j} V_{q_{0}}\left(c_{0}\left(\tau_{0}\right)\right) .
$$

Since $\Lambda=\mathbb{N}$ and $c_{j|Q|}(t) \in W_{q_{0}}$ for all $j \geq 0, t \in I_{j|Q|}$ we can assume without loss of generality that $c_{j|Q|}\left(\tau_{j|Q|}\right) \in$ $R_{e_{|Q|}}\left(G_{e_{|Q|}}\right) \cap W_{q_{0}}$. Lemma 1 guarantees that the interval $I_{j|Q|}$ has length $\tau_{j|Q|+1}-\tau_{j|Q|} \leq B_{q_{0}}\left(c_{j|Q|}\left(\tau_{j|Q|}\right)\right) / \gamma_{q_{0}}$. Thus we see the total time spent in $D_{q_{0}}$ is

$$
\begin{aligned}
\sum_{j=0}^{\infty}\left(\tau_{j|Q|+1}-\tau_{j|Q|}\right) & \leq \frac{1}{\gamma_{q_{0}}} \sum_{j=0}^{\infty} B_{q_{0}}\left(c_{j|Q|}\left(\tau_{j|Q|}\right)\right) \\
& \leq \frac{b}{\gamma_{q_{0}}}\left(V_{q_{0}}\left(c_{0}\left(\tau_{0}\right)\right)\right)^{a} \sum_{j=0}^{\infty}\left(r^{a}\right)^{j} \\
& <\infty,
\end{aligned}
$$

where second inequality follows from $\mathbf{C} 2$ and (5), and the final inequality follows since $r<1$ and $a>0$.

The same argument shows that the execution spends only a finite amount of time in domain $D_{q}$ for all $q \in Q$.

Corollary 1: Any execution $\chi$ with $\Lambda=\mathbb{N}$ and $c_{0}\left(\tau_{0}\right) \in$ $Z_{\rho(0)}$ converges to $\left\{z_{q}\right\}_{q \in Q}$.

\section{Simple Hybrid Mechanical Systems}

Mechanical systems undergoing impacts are naturally modeled as hybrid systems. In this section, we will consider hybrid systems of this form and demonstrate how one obtains such systems from hybrid Lagrangians, which are the hybrid analogue of Lagrangians. For more on hybrid Lagrangians and Lagrangian hybrid systems, see [12], [13] and [14].

Lagrangians. Consider a configuration space ${ }^{2} \Theta$ and a Lagrangian $L: T \Theta \rightarrow \mathbb{R}$ given in coordinates by:

$$
L(\theta, \dot{\theta})=\frac{1}{2} \dot{\theta}^{T} M(\theta) \dot{\theta}-U(\theta)
$$

where $M(\theta)$ is positive definite and symmetric and $U(\theta)$ is the potential energy. For the sake of simplicity, we assume $\Theta \subset \mathbb{R}^{n}$ since all our results are local, i.e., we can work within a coordinate chart. The equations of motion are then given in coordinates by the Euler-Lagrange equations:

$$
\frac{d}{d t} \frac{\partial L}{\partial \dot{\theta}}-\frac{\partial L}{\partial \theta}=0
$$

In the case of Lagrangians of the form given in (6), the Euler-Lagrange equations become:

$$
M(\theta) \ddot{\theta}+C(\theta, \dot{\theta}) \dot{\theta}+N(\theta)=0,
$$

where $C(\theta, \dot{\theta})$ is the Coriolis matrix and $N(\theta)=\frac{\partial U}{\partial \theta}(\theta)$. Setting $x=\left(\theta^{T}, \dot{\theta}^{T}\right)^{T}$, the Lagrangian vector field, $f_{L}$, associated to $L$ takes the familiar form

$$
\dot{x}=f_{L}(x)=\left(\begin{array}{c}
\dot{\theta} \\
M(\theta)^{-1}(-C(\theta, \dot{\theta}) \dot{\theta}-N(\theta))
\end{array}\right) .
$$

This process of associating a dynamical system to a Lagrangian will be mirrored in the setting of hybrid systems. First, we introduce the notion of a hybrid Lagrangian.

\footnotetext{
${ }^{2}$ Note that we denote the configuration space by $\Theta$ rather than $Q$, due to the fact that $Q$ denotes the vertices of the graph of a hybrid system.
}

Definition 6: A hybrid Lagrangian is a tuple, $\boldsymbol{L}=$ $(\Theta, L, h)$, where

- $\Theta \subset \mathbb{R}^{n}$ is the configuration space,

- $L: T \Theta \rightarrow \mathbb{R}$ is a Lagrangian of the form give in (6),

- $h: \Theta \rightarrow \mathbb{R}$ is a unilateral constraint function, where we assume that 0 is a regular value of $h$.

Domains from constraints. Given a smooth (unilateral constraint) function $h: \Theta \rightarrow \mathbb{R}$ on a configuration space $\Theta$ such that $h^{-1}(0)$ is a smooth manifold, i.e., 0 is a regular value of $h$, we can construct a domain and a guard explicitly. To this constraint function we have an associated domain, $D_{h}$, defined to be the manifold (with boundary):

$$
D_{h}=\{(\theta, \dot{\theta}) \in T \Theta: h(\theta) \geq 0\} .
$$

Similarly, we have an associated guard, $G_{h}$, defined as the following submanifold of $D_{h}$ :

$$
G_{h}=\left\{(\theta, \dot{\theta}) \in T \Theta: h(\theta)=0 \text { and } d h_{\theta} \dot{\theta} \leq 0\right\},
$$

where $d h(\theta)=\left(\begin{array}{lll}\frac{\partial h}{\partial \theta_{1}}(\theta) & \cdots & \frac{\partial h}{\partial \theta_{n}}(\theta)\end{array}\right)$. Note that the requirement that 0 is a regular value of $h$ is equivalent to requiring that $d h(\theta) \neq 0$ when $h(\theta)=0$.

Lagrangian Hybrid Systems. Given a hybrid Lagrangian $\mathbf{L}=(\Theta, L, h)$, the Lagrangian hybrid system associated to $\mathbf{L}$ is the hybrid system

$$
\mathscr{H}_{\mathbf{L}}=\left(\Gamma=(\{q\},\{(q, q)\}), D_{\mathbf{L}}, f_{\mathbf{L}}, G_{\mathbf{L}}, R_{\mathbf{L}}\right),
$$

where $D_{\mathbf{L}}=\left\{D_{h}\right\}, f_{\mathbf{L}}=\left\{f_{L}\right\}, G_{\mathbf{L}}=\left\{G_{h}\right\}$ and $R_{\mathbf{L}}=$ $\left\{R_{h}\right\}$ with $R_{h}(\theta, \dot{\theta})=(\theta, P(\theta, \dot{\theta}))$, where

$$
\begin{aligned}
& P(\theta, \dot{\theta})= \\
& \dot{\theta}-(1+e) \frac{d h(\theta) \dot{\theta}}{d h(\theta) M(\theta)^{-1} d h(\theta)^{T}} M(\theta)^{-1} d h(\theta)^{T} .
\end{aligned}
$$

Example 1: To provide an example of the concepts introduced in this paper, we will consider the hybrid system modeling a pendulum impacting the ground. This system has as its hybrid Lagrangian the tuple $\mathbf{L}_{\text {pend }}=\left(\mathbb{R}, L_{\text {pend }}, h_{\text {pend }}\right)$, where $L_{\text {pend }}(\theta, \dot{\theta})=\frac{1}{2} m l^{2} \dot{\theta}^{2}-m g l \sin (\theta)$ and $h_{\text {pend }}(\theta)=$ $\sin (\theta)$, with $\theta$ the angle of the pendulum from horizontal, $m$ the mass of the bob, $l$ the length of the rod and $g$ the acceleration due to gravity.

From the hybrid Lagrangian $\mathbf{L}_{\text {pend }}$, we obtain the hybrid model of the pendulum:

$\mathscr{H}_{\text {pend }}=\left(\Gamma=(\{q\},\{(q, q)\}), D_{\text {pend }}, G_{\text {pend }}, R_{\text {pend }}, F_{\text {pend }}\right)$, where $D_{\text {pend }}=\left\{D_{h_{\text {pend }}}\right\}, F_{\text {pend }}=\left\{f_{L_{\text {pend }}}\right\}, G_{\text {pend }}=$ $\left\{G_{h_{\text {pend }}}\right\}$ and $R_{\text {pend }}=\left\{R_{h_{\text {pend }}}\right\}$ with

$$
\begin{aligned}
D_{h_{\text {pend }}} & =\left\{\left(\begin{array}{c}
\theta \\
\dot{\theta}
\end{array}\right) \in \mathbb{R}^{2}: \sin (\theta) \geq 0\right\} \\
G_{h_{\text {pend }}} & =\left\{\left(\begin{array}{c}
\theta \\
\dot{\theta}
\end{array}\right) \in \mathbb{R}^{2}: \sin (\theta)=0 \text { and } \dot{\theta} \leq 0\right\} \\
R_{h_{\text {pend }}}(\theta, \dot{\theta}) & =\left(\begin{array}{c}
\theta \\
-e \dot{\theta}
\end{array}\right) \\
f_{L_{\text {pend }}}(\theta, \dot{\theta}) & =\left(\begin{array}{c}
\dot{\theta} \\
-\frac{g}{l} \cos (\theta)
\end{array}\right)
\end{aligned}
$$


where here $0 \leq e \leq 1$ is the coefficient of restitution.

We note that $(0,0)$ and $(\pi, 0)$ are isolated Zeno equilibria of $\mathscr{H}_{\text {pend }}$. Moreover, it is easy to verify through Theorem 2 that this system is Zeno, although we will use the results presented in the next section to establish this fact. Also note that this hybrid system is actually characteristic of Lagrangian hybrid systems that have isolated Zeno equilibria and are Zeno as will be seen in the next section.

\section{ZENO BEHAVIOR IN LAGRANGIAN HYBRID SYSTEMS}

In this section, we characterize Zeno behavior in Lagrangian hybrid systems with isolated Zeno equilibria. This characterization relies, in a fundamental fashion, on the following proposition.

Proposition 1: If the Lagrangian hybrid system $\mathscr{H}_{\mathbf{L}}$ has an isolated Zeno equilibrium, then

L1: $\Theta$ is one-dimensional.

L2: The Zeno equilibria takes the form

$$
z=\left\{\left(\begin{array}{c}
\theta^{*} \\
0
\end{array}\right)\right\}
$$

L3: $P(\theta, \dot{\theta})=-e \dot{\theta}$, where $0 \leq e \leq 1$ is the coefficient of restitution.

Proof: L1: If $z=\left\{\left(\theta^{*}, \dot{\theta}^{*}\right)^{T}\right\}$ is an isolated Zeno equilibrium of $\mathscr{H}_{L}$, then there exists a neighborhood $W$ of $\left(\theta^{*}, \dot{\theta}^{*}\right)^{T}$ that contains no equilibria of (7) and no other Zeno equilibria. It follows that that $\left(\theta^{*}, \dot{\theta}^{*}\right)^{T}$ is the unique fixed point of $R_{h}$ in $W$. Now for $(\theta, \dot{\theta}) \in G_{L}, h(\theta)=0$ and $d h(\theta) \neq 0$ so using the fact that $M(\theta)^{-1}$ is positive definite, it follows that:

$$
\begin{aligned}
R_{h}(\theta, \dot{\theta})=(\theta, \dot{\theta}) & \Longleftrightarrow(d h(\theta) \dot{\theta}) M(\theta)^{-1} d h(\theta)^{T}=0 \\
& \Longleftrightarrow d h(\theta) \dot{\theta}=0 .
\end{aligned}
$$

Therefore, if $\operatorname{dim}\left(T_{\theta} \Theta\right) \geq 2$ then $d h(\theta) \dot{\theta}=0$ defines a hyperplane in $T_{\theta} \Theta$ of points in the guard, each of which is a fixed point of $R_{h}$. Thus, when $\operatorname{dim}(\Theta) \geq 2$, there can be no isolated Zeno equilibria.

$\mathbf{L} 2$ and $\mathbf{L} 3$ follow from $\mathbf{L} 1$.

Notation 1: From this point on, we adopt the following notation: $d h(\theta)=h^{\prime}(\theta)$ and $\frac{\partial U}{\partial \theta}(\theta)=U^{\prime}(\theta)$, since we will be interested in $h$ and $U$ that are functions of one real variable as a result of Proposition 1.

A Characterization of Zeno Behavior. We have shown that hybrid Lagrangian systems with isolated Zeno equilibria are necessarily quite simple. The main result in this section gives an explicit characterization of isolated Zeno equilibria for Lagrangian hybrid systems. We note that if $z=\left\{\left(\theta^{*}, 0\right)^{T}\right\}$ is an isolated Zeno equilibrium of a Lagrangian hybrid system $\mathscr{H}_{\mathbf{L}}$, then we can set $\theta^{*}=0$ without loss of generality by translating coordinates.

Theorem 2: Let $\mathscr{H}_{\mathbf{L}}$ be a Lagrangian hybrid system. If $z=\{0\}$ is an isolated Zeno equilibrium of $\mathscr{H}_{\mathbf{L}}$, then there is a neighborhood $W$ of 0 such that for all $x \in W$ there exists a unique Zeno execution $\chi=(\Lambda, I, \rho, C)$ with $c_{0}\left(\tau_{0}\right)=x$ converging to $\{0\}$ if and only if
1) The coefficient of restitution $e<1$

2) $\operatorname{sign}\left(h^{\prime}(0)\right)=\operatorname{sign}\left(U^{\prime}(0)\right)$

Before proving this theorem, we note that the following functions:

$$
\begin{aligned}
V(\theta, \dot{\theta}) & =\frac{1}{2} M(\theta) \dot{\theta}^{2}+U(\theta)-U(0) \\
B(\theta, \dot{\theta}) & =\frac{M(0)}{U^{\prime}(0)} \dot{\theta}+\sqrt{\frac{M(0)^{2}}{U^{\prime}(0)^{2}} \dot{\theta}^{2}+2 \frac{M(0)}{U^{\prime}(0)}} \theta
\end{aligned}
$$

and constants:

$$
\begin{aligned}
a & =\frac{1}{2} \\
b & =2 \sqrt{2}\left|\frac{\sqrt{M(0)}}{U^{\prime}(0)}\right| \\
r & =e^{2}
\end{aligned}
$$

will be shown to satisfy the conditions in Theorem 1 , thus giving a proof of Zeno behavior in a neighborhood $Z$ of 0 .

Proof:

$(\Leftarrow)$ We show that when $\operatorname{sign}\left(h^{\prime}(0)\right)=\operatorname{sign}\left(U^{\prime}(0)\right)$ and $e<1$, the functions and constants as by equations (9)-(13), satisfy all the conditions of Theorem 1 .

First, since $f_{L}(0) \neq 0$ and $\{0\}$ is an isolated Zeno equilibrium, we can choose an open ball $W$ around 0 such that $W$ contains no other Zeno equilibria and $W$ contains no equilibria of $f_{L}$. Let $W_{1}$ be the projection of $W$ on the $\theta$-axis.

We will also repeatedly use the fact that elements in $\left(G_{h} \cup\right.$ $\left.R_{h}\left(G_{h}\right)\right) \cap W$ take the form $(0, \dot{\theta}) \in \mathbb{R}^{2}$. Indeed, if $(\theta, \dot{\theta})^{T} \in$ $G_{h} \cap W$, then $h(\theta)=0$, by definition of $G_{h}$. By $\mathbf{L} 3, h(\theta)=0$ imples that $(\theta, 0)^{T}$ is a Zeno equilibrium in the ball $W$. Therefore $\theta=0$ since, 0 is the only Zeno equilibrium in $W$. Elements of $R_{h}\left(G_{h}\right) \cap W$ have the same form by $\mathbf{L} 3$.

EC1: Proving that $V$ is positive on $W \backslash\{0\}$ is equivalent to showing that $U(\theta)>U(0)$ on $W_{1} \backslash\{0\}$. By taking the Taylor series expansion of $h(\theta)$ about $\theta=0$, it follows that $\operatorname{sign}(h(\theta))=\operatorname{sign}\left(h^{\prime}(0) \theta\right)$. Now, from the Taylor series expansion of $U$, we see that

$$
U(\theta)-U(0)=U^{\prime}(0) \theta+O\left(\theta^{2}\right) .
$$

Since we are assuming that $\operatorname{sign}\left(h^{\prime}(0)\right)=\operatorname{sign}\left(U^{\prime}(0)\right)$, $h(\theta)>0$ implies $h^{\prime}(0) \theta>0$ and thus $U^{\prime}(0) \theta>0$ on $W_{1} \backslash\{0\}$. So $U(\theta)-U(0)>0$ on $W_{1} \backslash\{0\}$ and EC1 holds.

EC2: Follows from conservation of energy.

EC3: Since $\Theta$ is one dimensional by $\mathbf{L} 1$, the vector field $f_{L}$ becomes:

$$
\left(\begin{array}{l}
\dot{\theta} \\
\ddot{\theta}
\end{array}\right)=f_{L}(\theta, \dot{\theta})=\left(\begin{array}{c}
\dot{\theta} \\
-\frac{1}{M(\theta)}\left(\frac{1}{2} M^{\prime}(\theta) \dot{\theta}^{2}+U^{\prime}(\theta)\right)
\end{array}\right) .
$$

Since this vector field has no equilibrium points:

$$
\ddot{\theta}(0,0)=-\frac{U^{\prime}(0)}{M(0)} \neq 0 .
$$


Moreover, $M(0)>0$ and $U^{\prime}(0) \theta \geq 0$ imply $\frac{M(0)}{U^{\prime}(0)} \theta \geq 0$.

So for $(\theta, \dot{\theta})^{T} \in W$, we have

$$
\sqrt{\frac{M(0)^{2}}{U^{\prime}(0)^{2}} \dot{\theta}^{2}+2 \frac{M(0)}{U^{\prime}(0)}} \theta \geq\left|\frac{M(0)}{U^{\prime}(0)} \dot{\theta}\right| .
$$

Therefore, defining $B$ as in equation (10), we have $B(\theta, \dot{\theta}) \geq$ 0 on $W$. Thus we have shown that EC3 holds.

EC4: Taking the time derivative of $B$, we see that

$$
\dot{B}(\theta, \dot{\theta})=\frac{M(0)}{U^{\prime}(0)} \ddot{\theta}+\frac{\frac{M(0)}{U^{\prime}(0)} \dot{\theta}}{\sqrt{\frac{M(0)^{2}}{U^{\prime}(0)^{2}} \dot{\theta}^{2}+2 \frac{M(0)}{U^{\prime}(0)}} \theta}\left(1+\frac{M(0)}{U^{\prime}(0)} \ddot{\theta}\right)
$$

From equation (16), it follows that

$$
\frac{M(0)}{U^{\prime}(0)} \ddot{\theta}(0,0)=-1 \text {. }
$$

Therefore $\dot{B}$ is continuous at $(0,0)^{T}$ and $\dot{B}(0,0)=-1$. Thus $\dot{B}(\theta, \dot{\theta})<0$ in $W$ and EC4 holds.

ED1 and C1: $V\left(R_{h}(0, \dot{\theta})\right)=e^{2} V(0, \dot{\theta})$, by $\mathbf{L} 3$ of Proposition 1 , and the definition of $V$.

$$
\begin{aligned}
\text { C2: For }(0, \dot{\theta})^{T} & \in R_{h}\left(G_{h}\right) \cap W, \\
B(0, \dot{\theta}) & =\frac{M(0)}{U^{\prime}(0)} \dot{\theta}+\sqrt{\left(\frac{M(0)}{U^{\prime}(0)} \dot{\theta}\right)^{2}} \\
& \leq 2\left|\frac{M(0)}{U^{\prime}(0)}\right||\dot{\theta}| \\
& =2 \sqrt{2}\left|\frac{\sqrt{M(0)}}{U^{\prime}(0)}\right| V(0, \dot{\theta})^{1 / 2} .
\end{aligned}
$$

Thus the relative size condition $\mathbf{C} 2$ holds with constants defined by equations (11), (12).

$(\Rightarrow)$ Suppose that there is a neighborhood $W$ of 0 such that for all $x \in W$ there exists a unique Zeno execution $\chi=(\Lambda, I, \rho, C)$ with $c_{0}\left(\tau_{0}\right)=x$ that converges to $\{0\}$.

$\operatorname{sign}\left(h^{\prime}(0)\right)=\operatorname{sign}\left(U^{\prime}(0)\right)$ : Assume that $\operatorname{sign}\left(h^{\prime}(0)\right) \neq$ $\operatorname{sign}\left(U^{\prime}(0)\right)$. Since $\{0\}$ is a Zeno equilibrium, $U^{\prime}(0) \neq 0$ by (16). Similar to the proof of EC $1, h(\theta)>0$ implies $h^{\prime}(0) \theta>$ 0 and $U^{\prime}(0) \theta<0$ near 0 . So given any neighborhood $W$ of 0 , there exists a neighborhood $N$ of 0 such that $N \times\{0\} \subset$ $W$ and for all $\theta \in N \backslash\{0\}, U(\theta)<U(0)$. If $\chi$ satisfies $c_{0}\left(\tau_{0}\right)=\left(\theta_{0}, 0\right)^{T} \in N \times\{0\}$, then $c_{i}(t)$ never reaches the open set

$$
\left\{(\theta, \dot{\theta})^{T}: V(\theta, \dot{\theta})>U\left(\theta_{0}\right)\right\}
$$

which contains the origin. Thus $\chi$ does not converge to the origin.

$e<1$ : Now assume that $\operatorname{sign}\left(h^{\prime}(0)\right)=\operatorname{sign}\left(U^{\prime}(0)\right)$ but $e=1$. Then there is a neighborhood $W$ of the origin such that $V(\theta, \dot{\theta})>0$ on $W \backslash\{0\}$ from the proof of EC1. If an execution $\chi$ has $c_{0}\left(\tau_{0}\right)=\left(\theta_{0}, \dot{\theta}_{0}\right) \in W \backslash\{0\}$, then $V\left(c_{i}(t)\right)=V\left(\theta_{0}, \dot{\theta}_{0}\right)$ for all $i \in \Lambda$ and $t \in I_{i}$. Thus $c_{i}(t)$ never reaches the open set

$$
\left\{(\theta, \dot{\theta})^{T}: V(\theta, \dot{\theta})<V\left(\theta_{0}, \dot{\theta}\right)\right\}
$$

which contains the origin. Thus $\chi$ does not converge to the origin.

Example 2: Consider the hybrid system $\mathscr{H}_{\text {pend }}$, defined in Example 1, modeling a pendulum impacting the ground. From the pendulum's Lagrangian, if follows that

$$
U_{\text {pend }}(\theta)=m g l \sin (\theta)=m g l\left(h_{\text {pend }}(\theta)\right),
$$

and so

$$
\operatorname{sign}\left(U_{\text {pend }}^{\prime}(0)\right)=\operatorname{sign}\left(h_{\text {pend }}^{\prime}(0)\right) .
$$

Therefore, the conditions of Theorem 2 hold if and only if the coefficient of restitution $e<1$. Thus, there is a neighborhood $W$ of $(0,0)^{T}$ such that for all $(\theta, \dot{\theta})^{T} \in W$, there is a Zeno execution $\chi=(\Lambda, I, \rho, C)$ with $c_{0}\left(\tau_{0}\right)=(\theta, \dot{\theta})$ converging to $\left\{(0,0)^{T}\right\}$ if and only if $e<1$.

An analogous statement holds for the Zeno equilibrium $\left\{(\pi, 0)^{T}\right\}$. As a result of Theorem 2, to prove that the pendulum is Zeno we only needed to evaluate two known functions at a single point.

\section{REFERENCES}

[1] S. Prajna, "Optimization-based methods for nonlinear and hybrid systems verification," Ph.D. dissertation, California Institute of Technology, 2005.

[2] A. Papachristodoulou and S. Prajna, "The construction of lyapunov functions using the sum of squares decomposition," in Proceedings of the 41st IEEE Conference on Decision and Control, 2002.

[3] S. Prajna and A. Jadbabaie, "Safety verification of hybrid systems using barrier certificates," in HSCC, 2004, pp. 477-492.

[4] M. Heymann, F. Lin, G. Meyer, and S. Resmerita, "Analysis of zeno behaviors in a class of hybrid systems," IEEE Transactions on Automatic Control, vol. 50, no. 3, pp. 376-384, 2005.

[5] A. D. Ames, P. Tabuada, and S. Sastry, On the Stability of Zeno Equilibria, lecture notes in com ed. Springer-Verlag, 2006, vol. 3927, pp. $34-48$.

[6] A. D. Ames and S. Sastry, "Characterization of Zeno behavior in hybrid systems using homological methods," in 24th American Control Conference, 2005.

[7] _ , "A homology theory for hybrid systems: Hybrid homology," in Hybrid Systems: Computation and Control, ser. Lecture Notes in Computer Science, M. Morari and L. Thiele, Eds., vol. 3414. Springer-Verlag, 2005, pp. 86-102.

[8] J. Zhang, K. H. Johansson, J. Lygeros, and S. Sastry, "Zeno hybrid systems," Int. J. Robust and Nonlinear Control, vol. 11, no. 2, pp. 435-451, 2001.

[9] M. K. Camlibel and J. M. Schumacher, "On the Zeno behavior of linear complementarity systems," in 40th IEEE Conference on Decision and Control, 2001.

[10] J. Shen and J.-S. Pang, "Linear complementarity systems: Zeno states," SIAM Journal on Control and Optimization, vol. 44, no. 3, pp. 10401066, 2005.

[11] A. D. Ames, A. Abate, and S. Sastry, "Sufficient conditions for the existence of Zeno behavior," ser. 44th IEEE Conference on Decision and Control and European Control Conference ECC, 2005.

[12] A. D. Ames, "A categorical theory of hybrid systems," Ph.D. dissertation, University of California, Berkeley, 2006.

[13] A. D. Ames and S. Sastry, "Routhian reduction of hybrid lagrangians and lagrangian hybrid systems," in Submitted to the American Control Conference, 2006.

[14] A. D. Ames, H. Zheng, R. D. Gregg, and S. Sastry, "Is there life after Zeno? Taking executions past the breaking (Zeno) point," submitted to the 2006 American Control Conference.

[15] B. Brogliato, Nonsmooth Mechanics. Springer-Verlag, 1999.

[16] S. Simic, K. H. Johansson, S. Sastry, and J. Lygeros, "Towards a geometric theory of hybrid systems," in $H S C C$, ser. LNCS, B. Krogh and N. Lynch, Eds., vol. 1790. Springer Verlag, 2000, pp. 421-436. 